\title{
Association between contrast-enhanced ultrasonography and histopathological findings of the metastatic lymph nodes of patients with head and neck cancer: A preliminary study
}

\author{
SHIN-ICHI OIKAWA ${ }^{1}$, KIYOTO SHIGA ${ }^{1}$, DAISUKE SAITO ${ }^{1}$, KATSUNORI KATAGIRI $^{1}$, AYA IKEDA ${ }^{1}$, \\ KODAI TUCHIDA ${ }^{1}$, JUN MIYAGUCHI ${ }^{1}$, KAZUYUKI ISHIDA ${ }^{2}$ and TAMOTSU SUGAI ${ }^{2}$ \\ Departments of ${ }^{1} \mathrm{Head}$ and Neck Surgery and ${ }^{2}$ Molecular Diagnostic Pathology, Iwate \\ Medical University School of Medicine, Morioka, Iwate 020-8505, Japan
}

Received September 11, 2017; Accepted November 20, 2017

DOI: $10.3892 / \mathrm{ol} .2018 .7835$

\begin{abstract}
The present study aimed to investigate the usefulness of contrast-enhanced ultrasonography (CEUS) and a newly developed analysis software for the detection of microcapillary network distribution in lymph nodes of patients with head and neck cancer (HNC) by comparing the CEUS and histopathological findings. Patients that were diagnosed with HNC between February and September 2016 were enrolled. A total of five patients underwent resection of the primary tumor and neck dissection as their initial treatment. The cervical lymph nodes of these patients were analyzed by CEUS intraoperatively, and their surgical specimens were examined histopathologically. The patients were diagnosed using a combination of physical examination, computed tomography, magnetic resonance imaging and fluorodeoxyglucose-positron emission tomography. For CEUS examination, the microbubble contrast agent Sonazoid ${ }^{\mathrm{TM}}$ was injected into a peripheral vein. Video images of the metastatic lymph nodes were captured, and these were subjected to analysis by a newly developed image-analysis software. It was possible to perform intraoperative CEUS of metastatic lymph nodes and obtain accurate matched sections for histopathological examination. Hematoxylin and eosin and cluster of differentiation (CD)34 staining revealed that the software was able to accurately detect capillary vessels in metastatic lymph nodes. However, a number of perfusion deficits were observed in these lymph nodes. In conclusion, by using CEUS with the analysis software, the density and distribution of blood vessels in the metastatic lymph nodes of patients with HNC was revealed.
\end{abstract}

Correspondence to: Dr Kiyoto Shiga, Department of Head and Neck Surgery, Iwate Medical University School of Medicine, 19-1 Uchimaru, Morioka, Iwate 020-8505, Japan

E-mail:kshiga@iwate-med.ac.jp

Key words: contrast-enhanced ultrasonography, Sonazoid ${ }^{\mathrm{TM}}$, head and neck cancer, metastatic lymph node, histopathological findings, cluster of differentiation 34
Although the present study was limited and preliminary, it was concluded that this method may be useful to evaluate and to map the capillary vessels in the metastatic lymph nodes of patients with HNC.

\section{Introduction}

In order to improve the prognosis of patients with head and neck cancer (HNC), it is essential to accurately diagnose cervical lymph node metastases prior to the initiation of treatment $(1,2)$. Although computed tomography (CT), magnetic resonance imaging (MRI) and positron emission tomography (PET) may be used to identify metastatic lymph nodes, the nodes can be difficult to detect, particularly those that measure $<10 \mathrm{~mm}$ in diameter (2). Ultrasonography (US) may be used to evaluate lymph nodes that are $<10 \mathrm{~mm}$ in diameter. The clinical criteria currently being used to diagnose metastatic cervical lymph nodes by ultrasonography are an increase in nodal size and cortical thickness, a change in nodal shape, infiltration of surrounding structures, the presence of inhomogeneous internal echo patterns (including necrosis), the absence of echo-rich hilar structures and extracapsular spread $(3,4)$.

It has previously been demonstrated that lymph nodes undergoing metastatic change experience an increase in blood vessel volume and density prior to experiencing a change in nodal size (5). Ultrasonic color Doppler imaging is able to detect blood flow in and around lymph nodes but is unable to delineate the blood vessels themselves (6).

Sonazoid $^{\mathrm{TM}}$, a lipid-stabilized suspension of perfluorocarbon microbubbles, was used in the present study as an ultrasound contrast agent. In Japan, contrast-enhanced ultrasonography (CEUS) with Sonazoid ${ }^{\mathrm{TM}}$ is used for the evaluation of liver and mammary gland tumors $(7,8)$. It was hypothesized that it would also be possible to evaluate changes in lymph node blood vessel volume and density using CEUS with Sonazoid ${ }^{\mathrm{TM}}$. The objective of the present study was to evaluate the usefulness of CEUS with Sonazoid ${ }^{\mathrm{TM}}$ for identifying metastatic cervical lymph nodes in patients with HNC. Since enhancement of lymph nodes occurs so rapidly and the time to peak is very short, software capable of analyzing CEUS images of lymph nodes did not previously exist. Thus, novel analysis 
software was developed by the authors of the present study (9), and its usefulness was evaluated in the present study. Another problem faced was that adjustment of the scanning plane during evaluation of the head and neck region was difficult. This also occurred during evaluation of the sectioned surfaces of the surgical specimens, although to a lesser degree. A novel technique will be required to improve visualization of these areas. In the present study, intra-operative US was performed to assist in the acquisition of matched samples used for CEUS and histopathological examination. The association between images obtained via CEUS and histopathology performed on surgical specimens taken from metastatic cervical lymph nodes was evaluated.

\section{Materials and methods}

Patients. Patients with histologically proven head and neck squamous cell carcinoma who were treated at Iwate Medical University Hospital (Morioka, Japan) between February and June 2016 were enrolled in the present study. Patients with untreated lymph node metastasis diagnosed by a combination of physical examination, CT scan, MRI and fluorodeoxyglucose (FDG)-PET scan met the inclusion criterion. A total of five patients underwent surgery including neck dissection.

The present study was approved by the review board of Iwate Medical University Hospital and written informed consent was obtained from each patient. All procedures followed were in accordance with the ethical standards of The Responsible Committee on Human Experimentation (institutional and national) and with The Declaration of Helsinki (1975), as revised in 2013 (10).

Among the five patients, primary tumors were detected in the oral cavity in two patients. Primary tumors were also detected in the hypopharynx for two patients and in the larynx for one patient (Table I). The N-classification was N1 for one patient and N2b for four of the patients, according to the TNM staging system (11). The ages of the patients ranged from 65 to 75 years (mean, 71.0 years, median, 72 years), and all patients were male (Table I).

Ultrasound imaging. Conventional US and CEUS were performed using a LOGIQ E9 general imaging system (GE Healthcare, Chicago, IL, USA) and a high-frequency probe (6-15 MHz) for the evaluation of the cervical lymph nodes. In each patient, one cervical lymph node suspected of having metastatic disease was selected as the lymph node of interest prior to surgery. During neck dissection, the probe was placed directly on the lymph node of interest and B-mode data were obtained. Sonazoid $^{\text {TM }}$ was then injected into a peripheral vein, and the probe was replaced. Video was obtained from the hard disc drive of LOGIQ E9 for $\sim 1$ min following administration of the contrast agent. During the ultrasonographic examination, the mechanical index was set at 0.21 , and the frame rate at 19 frames per sec. The focus was on the lymph node of interest. The imaging conditions were kept constant. The examinations using B-mode ultrasound and CEUS were performed by the same investigator, and all data were digitally recorded. The lymph nodes of interest were analyzed using CEUS intraoperatively, and surgical specimens obtained from these nodes were examined histopathologically. The lymph nodes were carefully removed to allow an accurate match between the cut surface of the surgical specimen and the section examined via ultrasound.

Contrast agent for ultrasonography. Sonazoid ${ }^{\mathrm{TM}}$ (Daiichi-Sankyo, Co., Ltd., Tokyo, Japan) is a lyophilized preparation containing $16 \mu \mathrm{l}$ perfluorobutane microbubbles per vial that are reconstituted for injection. The contents of each vial were resuspended in $2 \mathrm{ml}$ of sterile water for injection. For patients with liver or mammary gland tumors, a single injection of $0.12 \mu \mathrm{MB} / \mathrm{kg}$ of the reconstituted suspension was administered in a cubital vein. Previous research has identified that similar enhancement effects can be obtained in cases of metastatic HNC involving the cervical lymph nodes using a dose of $0.012 \mu \mathrm{MB} / \mathrm{kg}$ (data not shown). In the present study, an initial dose of $0.012 \mu \mathrm{MB} / \mathrm{kg}$ was administered via a cubital vein intraoperatively from an $18 \mathrm{G}$ needle followed by $10 \mathrm{ml}$ saline flush.

Analysis software. A novel image-analysis software (IwmUltrasonic; version 2.0; Ditect Co., Ltd., Tokyo, Japan) was developed based on an algorithm previously reported (9). This software analyzes the video files obtained with CEUS. Visible particles were used to draw accumulated color points revealing the distribution of capillary vessels. This software is able to simultaneously calculate the density of particles in the drawn figures, which is hypothesized to represent the capillary density of the lymph nodes.

Histopathological examination. Tissues were fixed in $10 \%$ formalin at room temperature and embedded in paraffin wax. Sections were cut from the paraffin blocks to a $3 \mu \mathrm{m}$ thickness and mounted on poly-Llysine-coated glass slides (Matsunami Glass Ind., Ltd., Tokyo, Japan). Paraffin sections $(3-\mu \mathrm{m})$ were routinely dewaxed at $37^{\circ} \mathrm{C}$ for $7 \mathrm{~h}$ and rehydrated using $70-100 \%$ alcohol solution $(70 ; 90 ; 95 ; 100 ; 100 ; 100 \%), 90 \mathrm{~min}$ for each interval according to routine procedures (12). The first sections were stained at room temperature for $40 \mathrm{~min}$ using hematoxylin and eosin (100\% Carrazi's hematoxylin and $0.5 \%$ eosin solution). The second sections were stained with mouse anti-CD34 monoclonal antibody (cat. no., IR632; ready-to-use; Dako; Agilent Technologies, Inc., Santa Clara, CA, USA) in order to make a comparison between morphology and CD34 immunoreactivity, then subjected to heat-induced epitope retrieval in High pH Target Retrieval solution (Dako; Agilent Technologies, Inc.). The slides were placed in $0.03 \%$ peroxidase-blocking reagent (Dako; Agilent Technologies, Inc.) at room temperature for $5 \mathrm{~min}$ to inhibit non-specific binding. Following primary antibody incubation at $97^{\circ} \mathrm{C}$ for $20 \mathrm{~min}$ (mouse anti-CD34 monoclonal antibody; dilution, ready-to-use; cat. no. IR632; Dako; Agilent Technologies, Inc.), sections were analyzed using the EnVision ${ }^{\mathrm{TM}}$ HRP detection system (cat. no. K8000, Dako; Agilent Technologies, Inc.) according to the protocol of the manufacturer. The antigen-antibody complex was visualized with DAB+ liquid chromogen (Dako; Agilent Technologies, Inc.) and counterstained at room temperature for 2 min with $100 \%$ Carrazi's hematoxylin prior to mounting.

\section{Results}

A newly developed image-analysis software was utilized to construct images from a microbubble contrast agent, allowing 
Table I. Profiles of the patients and lymph nodes of interest.

\begin{tabular}{lllllllc}
\hline Patient & Age & Gender & $\begin{array}{c}\text { Primary } \\
\text { tumor }\end{array}$ & Stage & $\begin{array}{c}\text { Lymph node } \\
\text { of interest }\end{array}$ & $\begin{array}{c}\text { Major } \\
\text { axis, mm }\end{array}$ & $\begin{array}{c}\text { Minor } \\
\text { axis, mm }\end{array}$ \\
\hline 1 & 71 & M & Larynx & T2N2bM0 & Left II & 15 & 11 \\
2 & 75 & M & Hypopharynx & T3N2bM0 & Left III & 24 & 21 \\
3 & 72 & M & Hypopharyn & T4aN2bM0 & Right III & 20 & 11 \\
4 & 65 & M & Tongue & rN1 & Left II & 31 & 31 \\
5 & 72 & M & Floor of the mouth & TN2bM0 & Left I & 31 & 10 \\
\hline
\end{tabular}

${ }^{a}$ Union for international cancer control tumor node metastasis (11); M, male.

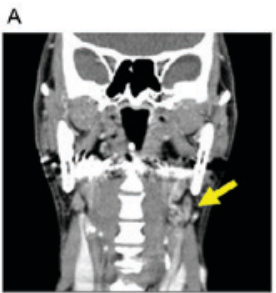

$\mathrm{E}$

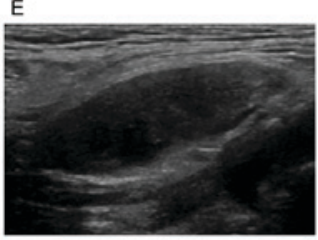

1
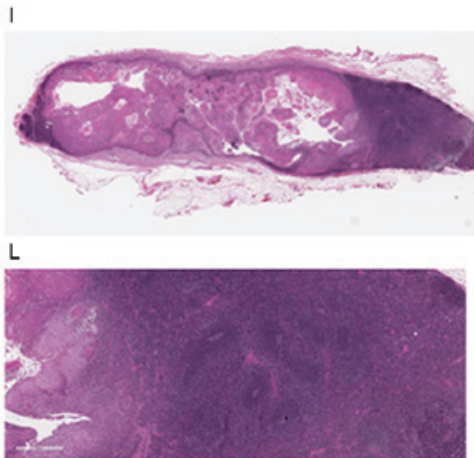

B

$\mathrm{F}$
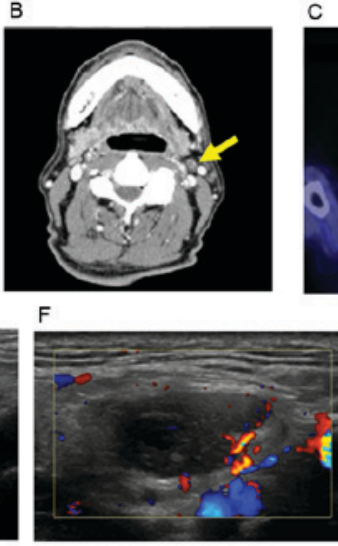

$\mathrm{J}$

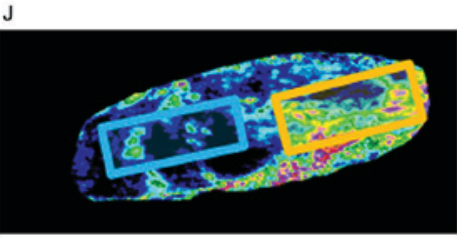

M

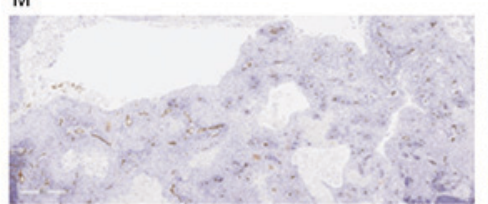

C

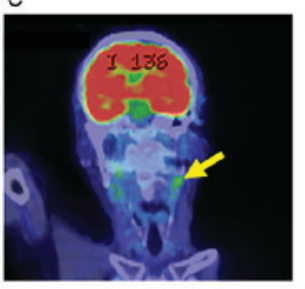

G

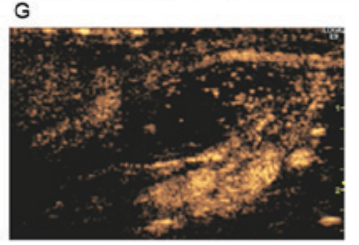

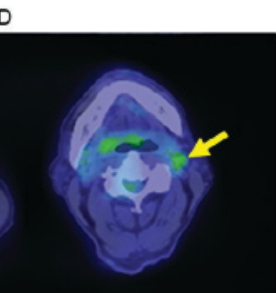

$\mathrm{H}$

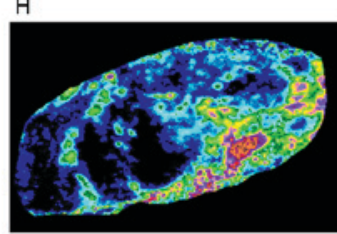

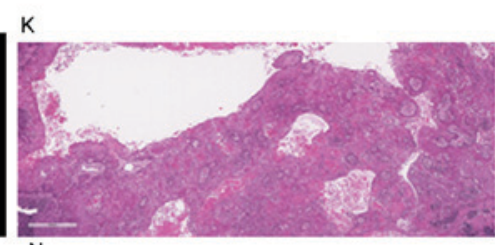

$\mathrm{N}$

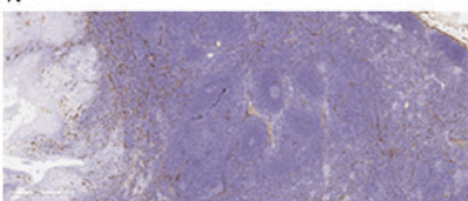

Figure 1. Histopathological findings, clinical and ultrasound images of case 5. Enhanced CT images in the (A) coronal view and (B) axial view of the lymph node of interest. Fluorodeoxyglucose-positron emission tomography/CT images in the (C) coronal view and (D) axial view of the lymph node of interest. The yellow arrows indicate the lymph node of interest. (E) Ultrasound B-mode image of US including the major axis of the lymph node of interest. (F) Color Doppler image of the lymph node of interest. (G) CEUS and (H) analyzed CEUS images of the lymph node of interest. (I) H\&E staining of the lymph node of interest including the major axis. Scale bar $=4 \mathrm{~mm}$. (J) The same image as H indicating the selected area of interest. Blue square, area of low contrast enhancement; orange square, area of high contrast enhancement. H\&E staining of (K) area of low contrast enhancement and (L) area of high contrast enhancement. CD34 immunohistochemical staining of $(\mathrm{M})$ area of low contrast enhancement and $(\mathrm{N})$ area of high contrast enhancement. Original magnification, $\mathrm{x} 25$ and scale bars $=800 \mathrm{~mm}(\mathrm{~K}, \mathrm{~L}, \mathrm{M}$, and N). CT, computed tomography; CEUS, contrast enhanced ultrasonography; CD34, cluster of differentiation 34; H\&E, hematoxylin and eosin.

visualization of vessel distribution and density in metastatic lymph nodes from patients with HNC. A typical result is presented in Fig. 1.

First, the internal structures of the metastatic cervical lymph nodes of the patients with HNC were compared. A total of 5 patients who underwent tumor resection with concomitant neck dissection as an initial treatment and whose lymph nodes were diagnosed as metastatic using CT, MRI or PET were enrolled in the present study. The profiles of the patients are presented in Table I. During neck dissection, video files of the metastatic lymph nodes were obtained using CEUS, and these images were subjected to analysis.

Typical results are presented in Fig. 1. Contrast-enhanced CT images (Fig. 1A and B) and FDG-PET images (Fig. 1C and D) identified a candidate lymph node in case 5. Ultrasound images obtained from the same lymph node using B-mode ultrasound, color Doppler, CEUS and CEUS with analysis software can be compared in Fig. 1E-H. In the B-mode image (Fig. 1E), the fatty hilum and internal structure of the lymph node are not clear. The CEUS mode image obtained using a 
Table II. Histopathological and ultrasonography image data of the lymph nodes.

\begin{tabular}{lccccc}
\hline Patient & Necrosis & $\begin{array}{c}\text { Normal lymph } \\
\text { follicles }\end{array}$ & $\begin{array}{c}\text { Color Doppler imaging- } \\
\text { matched capillary vessels }\end{array}$ & $\begin{array}{c}\text { CEUS-matched } \\
\text { capillary vessels }\end{array}$ & $\begin{array}{c}\text { Perfusion } \\
\text { defect }\end{array}$ \\
\hline 1 & - & + & + & ++ & ++ \\
2 & - & - & - & + & + \\
3 & + & - & + & ++ & + \\
4 & +++ & + & + & ++ & ++ \\
5 & + & - & + & + & + \\
\hline
\end{tabular}

Necrosis: -; none, + ; observed in under $20 \%$ of areas, ++ ; observed in 20 50\% of areas, +++ ; observed in over $50 \%$ of areas. Normal lymph follicles: -; none, +; observed, Color Doppler imaging-matched capillary vessels; CEUS-matched capillary vessels: -; none, +; only thick vessels were detected, ++ ; thick vessels and peripheral thin vessels were detected. Perfusion defect: -; none, + ; observed in $<50 \%$ of areas, ++ ; observed in $\geq 50 \%$ of areas, except for necrotic areas. CEUS, contrast-enhanced ultrasonography.

microbubble contrast agent is presented in Fig. 1G, and the CEUS mode image analyzed by the software is shown in Fig. $1 \mathrm{H}$. A comparison between Fig. $1 \mathrm{~F}$ and $\mathrm{H}$ identifies that color Doppler provides only a rough sketch of the capillary vessels present in lymph nodes, whereas the analyzed CEUS images depicts micro-vascular patterns in the lymph node in greater detail. Fig. 1I demonstrates the H\&E pattern of the plane including the major axis of the lymph node of interest. In Fig. 1J, an area of high contrast enhancement (orange square) and an area of low contrast enhancement (blue square) were selected, and these areas (Fig. 1K-N) were examined by $\mathrm{H} \& \mathrm{E}$ (Fig. 1K and L) and CD34 staining (Fig. 1M and N). As indicated in Fig. 1L and N, the normal lymph follicles were preserved, and around these lymph follicles thick capillary vessels were present. Thin capillary vessels were prevalent in the border of the tumor and normal lymph follicles. There were several thin capillary vessels including a few with no lumen in the area of low contrast enhancement (Fig. 1K and M).

Histopathological images of lymph nodes and analyzed CEUS images from cases 1-4 are presented in Fig. 2, and the characteristic features of these metastatic lymph nodes are presented in Table II. Micronecroses were observed in the lymph nodes from cases 3 and 5, and major necrosis was observed in the lymph node from case 4 . Non-metastatic normal lymph follicles were observed in cases 1 and 5 . Although histopathological examination confirmed the presence of capillary vessels in lymph nodes, color Doppler imaging was not able to detect them in two cases. The analyzed CEUS image was successful in detecting those same capillary vessels. However, a number of perfusion deficits were observed in analyzed CEUS images of lymph nodes, suggesting the presence of perfusion blocking factors in metastatic lymph node tissue.

\section{Discussion}

Metastasis to cervical lymph nodes has previously been identified using imaging modalities including CT, MRI and FDG-PET. However, the diagnostic power of these imaging modalities is limited when the lymph node is small, particularly when the diameter is $<10 \mathrm{~mm}$ and when characteristic features including necrosis and calcification are missing (13).
Ultrasound is readily available and its power of resolution is greater compared with both CT and MRI (3).

Ultrasound examination used to identify cervical lymph node metastasis in patients with HNC is able to detect a shift in or disappearance of the hilum of lymph nodes, an increase in size of the nodes and central necrosis $(3,4)$. However, no specific diagnostic criteria have been established. Color Doppler imaging is used to detect vessel flow in and around lymph nodes, but its accuracy has not been investigated. In the present study, the accuracy of color Doppler imaging was identified to be inferior compared with CEUS imaging (Fig. 1).

In mouse models, it has been reported that an increase in blood vessel volume and density precedes an increase in lymph node size in the early stages of lymph node metastasis (5). It has been hypothesized that detection of these changes by CEUS may allow early diagnosis of lymph node metastasis. This information may be applied to early detection of cervical lymph node metastasis in patients with HNC. Metastatic lymph nodes were examined in patients with advanced stages of the disease (Table I and Fig. 1). To allow accurate comparison of the cut surface of the surgical specimen with the section examined via ultrasound, intra-operative CEUS examination of the lymph node of interest was performed. Although a number of studies have evaluated intraoperative ultrasonography of the head and neck, the majority of them involved transoral surgery (14-16). A number of studies have investigated intraoperative evaluation of the cervical lymph nodes $(17,18)$. One of these studies (18) reported the usefulness of intraoperative US for patients with thyroid cancer, and the surgical method used was similar to the method used in the present study. To the best of our knowledge, the present study is the first to describe intraoperative US and CEUS during neck dissection in patients with HNC.

Regarding the accuracy of matching analyzed CEUS images with histopathological images using HE staining, matching was successful in the majority of cases, and the almost matched patterns are presented in Figs. 1 and 2. These data indicate that intraoperative US and CEUS are useful techniques for the acquisition of accurate images for US and histopathological examination. However, there was a defect on the upper side of the analyzed CEUS image in case 2. Perhaps this phenomenon was due to the direct touch of the ultrasound 
A

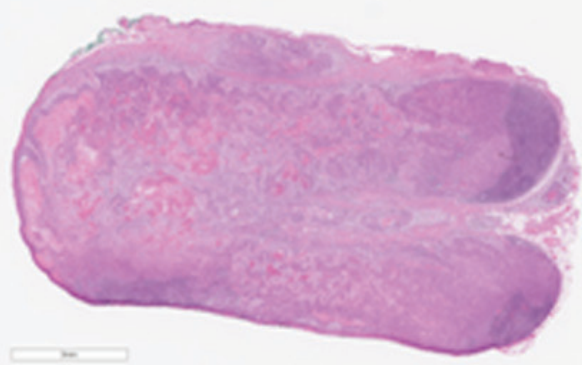

\section{c}

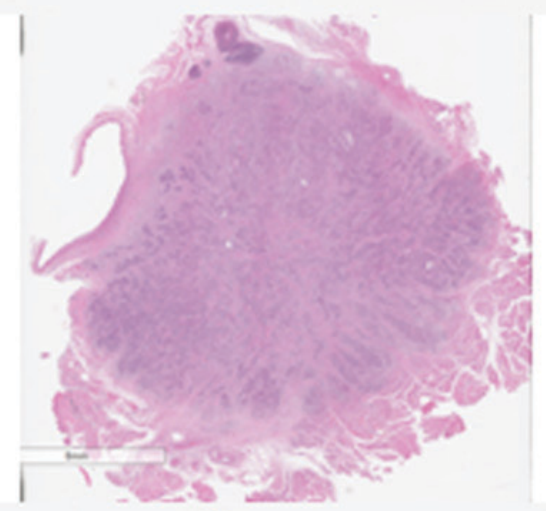

E
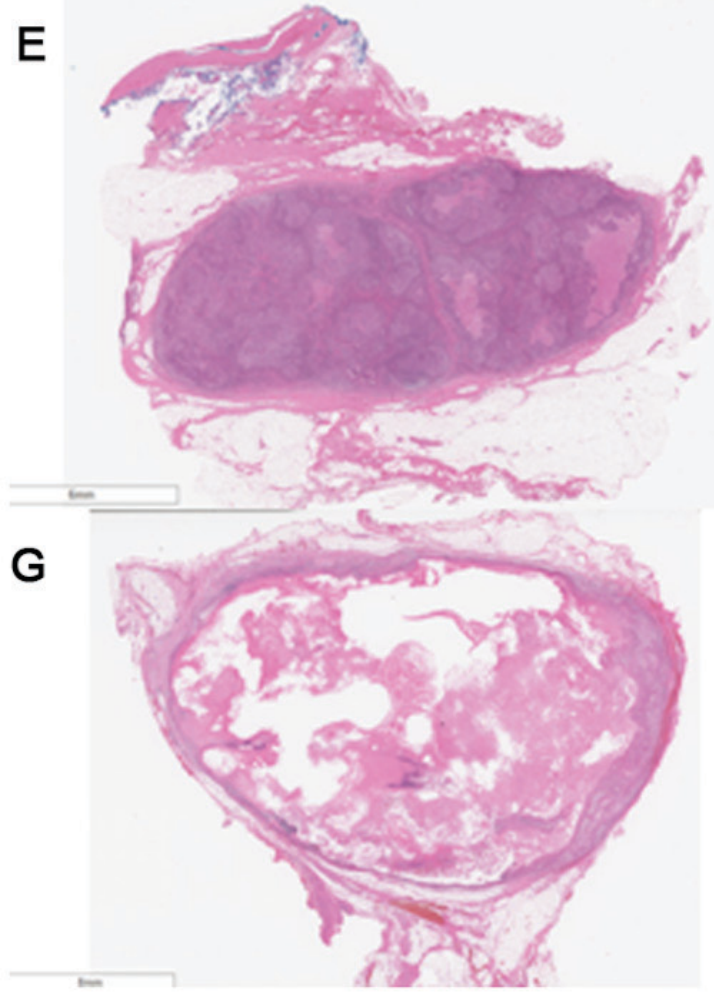

B

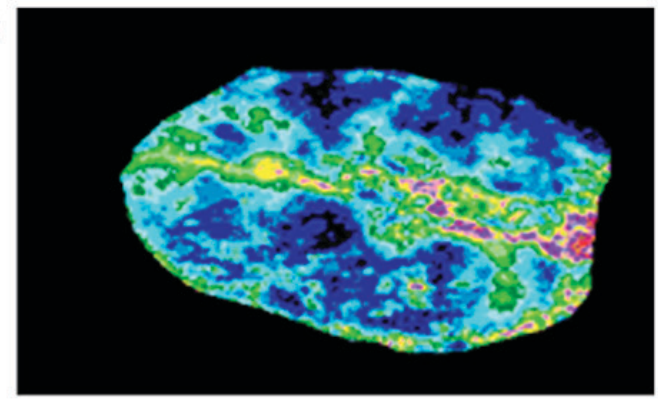

D

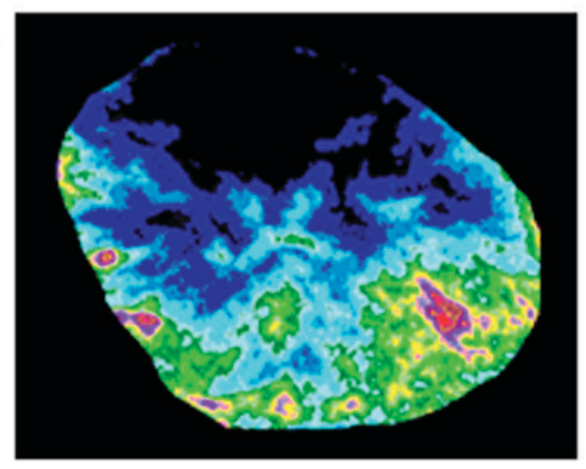

F

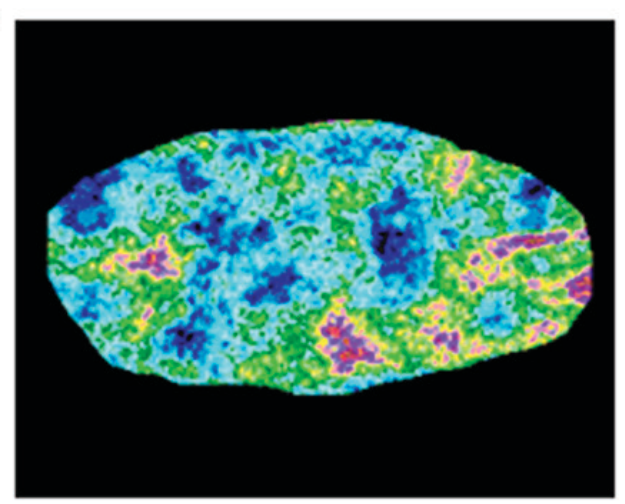

H

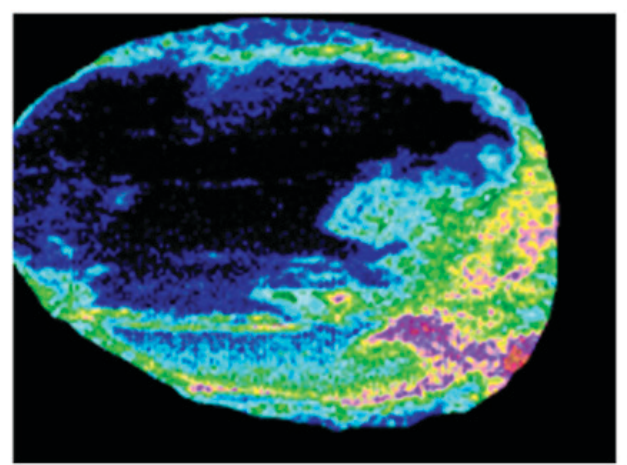

Figure 2. Histopathological and analyzed CEUS images of the metastatic lymph nodes of patients. (A) H\&E staining of metastatic lymph node section of case 1. (B) Analyzed CEUS image of case 1. (C) H\&E staining of metastatic lymph node section of case 2. (D) Analyzed CEUS image of case 2. (E) H\&E staining of metastatic lymph node section of case 3. (F) Analyzed CEUS image of case 3. (G) H\&E staining of metastatic lymph node section of case 4. (H) Analyzed CEUS image of case 4. H\&E, hematoxylin and eosin; CEUS, contrast enhanced ultrasonography. Scale bar in A, C, E, and G=4 mm.

probe to the surface of the lymph node. To avoid these image defects, an acoustic coupler could be used for acquisition of the ultrasound data.

Although there have been several reports that describe the usefulness of CEUS in the detection of metastatic axillary lymph nodes in patients with breast cancer $(19,20)$, there have been a limited number of reports concerning the use of CEUS for the identification of metastatic cervical lymph nodes $(21,22)$. The use of CEUS combined with the analysis software reveals the distribution of blood vessels in metastatic cervical lymph nodes in patients with HNC. A comparison of CEUS images and histopathological patterns identifies that the use of CEUS combined with software reveals a more detailed pattern of capillary vessels in metastatic lymph nodes compared with color Doppler. Color Doppler imaging provides only a rough sketch of the capillary vessels present in lymph nodes. 
However, although CEUS revealed vascular images in lymph nodes that were almost proportional to the histopathological vascular patterns, there were a number of perfusion deficits at CD34 stain positive areas. This indicated the presence of immature capillary vessels accompanying tumor infiltration of the cervical lymph nodes. Although there were regular capillary vessels in non-affected regions of the lymph node around the lymph follicles, capillary vessels were rich in the border of the tumor and lymph follicles (Fig. 1L and N). Additionally, when CEUS was employed for normal lymph nodes, capillary vessels were detected mainly in the hilum of the lymph nodes, and peripheral flow from the hilum was detected (data not shown). This was consistent with the histopathological findings of the distribution of capillary vessels in normal lymph nodes.

These results suggest that CEUS combined with the usage of the image analysis software was useful in the visualization of capillary distribution and density in the cervical lymph nodes of patients with HNC. Histopathological examination suggested that this may be used as a novel tool to analyze and classify the internal structure of metastatic lymph nodes prior to surgery or chemoradiotherapy. However, due to the limited number of patients in the present study, the association between the internal structure of the metastatic lymph nodes and the clinical characteristics and prognoses of patients with $\mathrm{HNC}$ remains unclear. Also as this study included only squamous cell carcinoma, other types of head and neck malignancies including adenocarcinoma of the salivary gland origin remain uninvestigated.

To conclude, intraoperative CEUS combined with novel analysis software, intraoperative US was able to reveal the distribution of blood vessels in the metastatic lymph nodes of patients with HNC. Although this is a preliminary study, the results indicated that the use of CEUS combined with software was able to reveal a more detailed pattern of capillary vessels in metastatic lymph nodes than color Doppler when these images were compared to histopathological examination. Further investigation with a larger number of samples from patients with $\mathrm{HNC}$ is required to clarify the association between CEUS images and histopathological patterns, as a number of perfusion defects were noted in CD34 stain positive areas.

\section{Acknowledgements}

The present study was supported by a KAKENHI grant-in-aid from the Japan Society for the Promotion of Science (grant no. 26462619) and Health Labour Sciences Research Grant.

\section{References}

1. Schuller D, McGuirt WF, McCabe BF and Young D: The prognostic significance of metastatic cervical lymph nodes. Laryngoscope 90: 557-570, 1980.

2. Spiro RH, Alfonso AE, Farr HW and Strong EW: Cervical node metastasis from epidermoid carcinoma of the oral cavity and oropharynx. A critical assessment of current staging. Am J Surg 128: 562-567, 1974.

3. Sumi M, Ohki M and Nakamura T: Comparison of sonography and $\mathrm{CT}$ for differentiating benign from malignant cervical lymph nodes in patients with squamous cell carcinoma of the head and neck. AJR Am J Roentgenol 176: 1019-1024, 2001.

4. Furukawa MK and Furukawa MK: Diagnosis of lymph node metastasis of head and neck cancer and evaluation of effects of chemoradiotherapy using ultrasonography. J Clin Oncol 15: 23-32, 2010.
5. Li L, Mori S, Kodama M, Sakamoto M, Takahashi S and Kodama T: Enhanced sonographic imaging to diagnose lymph node metastasis: Importance of blood vessel volume and density. Cancer Res 73: 2082-2092, 2013.

6. Giovagnorio F, Galluzzo M, Andreoli C, De CM and David V: Color Doppler sonography in the evaluation of superficial lymphomatous lymph nodes. J Ultrasound Med 21: 403-408, 2002.

7. Watanabe R, Matsumura M, Munemasa T, Fujimaki M and Suematsu M: Mechanism of hepatic parenchyma-specific contrast of microbubble-based contrast agent for ultrasonography: Microscopic studies in rat liver. Invest Radiol 42: 643-651, 2007.

8. Masumoto N, Kadoya T, Amioka A, Kajitani K, Shigematsu H, Emi A, Matsuura K, Arihiro K and Okada M: Evaluation of malignancy grade of breast cancer using perflubutane-enhanced ultrasonography. Ultrasound Med Biol 42: 1049-1057, 2016.

9. Ito K, Noro K, Yanagisawa Y, Sakamoto M, Mori S, Shiga K, Kodama T and Aoki T: High-accuracy ultrasound contrast agent detection method for diagnostic ultrasound imaging systems. Ultrasound Med Biol 41: 3120-3130, 2015.

10. World Medical Association: World Medical Association Declaration of Helsinki: Ethical principles for medical research involving human subjects. JAMA 310: 2191-2194, 2013.

11. Sobin LH, Gospodarowicz MK, Wittekind C (eds): TNM classification of malignant tumors, seventh edition. Blackwell Publishing Ltd, 2009.

12. Sugai T, Inomata M, Uesugi N, Jiao YF, Endoh M, Orii S and Nakamura S: Analysis of mucin, p53 protein and Ki-67 expressions in gastric differentiated-type intramucosal neoplastic lesions obtained from endoscopic mucosal resection samples: A proposal for a new classification of intramucosal neoplastic lesions based on nuclear atypia. Pathol Int 54: 425-435, 2004.

13. Curtin HD, Ishwaran H, Mancuso AA, Dalley RW, Caudry DJ and McNeil BJ: Comparison of CT and MR imaging in staging of neck metastases. Radiology 207: 123-130, 1998.

14. Andrews GA, Kwon M, Clayman G, Edeiken B and Kupferman ME: Technical refinement of ultrasound-guided transoral resection of pharyngeal/retropharyngeal thyroid carcinoma metastases. Head Neck 33: 166-170, 2011.

15. Goepfert RP, Liu C and Ryan WR: Trans-oral robotic surgery and surgeon-performed trans-oral ultrasound for intraoperative location and excision of an isolated retropharyngeal lymph node metastasis of papillary thyroid carcinoma. Am J Otolaryngo 36: 710-714, 2015.

16. Clayburgh DR, Byrd JK, Bonfili J and Duvvuri U: Intraoperative ultrasonography during transoral robotic surgery. Ann Otol Rhinol Laryngol 125: 37-42, 2016.

17. Al-lami A, Riffat F, Alamgir F, Dwivedi R, Berman L, Fish B and Jani O: Utility of an intraoperative ultrasound in lateral approach mini-parathyroidectomy with discordant pre-operative imaging. Eur Arch Otorhinolaryngo 270: 1903-1908, 2013.

18. Ertas B, Kaya H, Kurtulumus N, Yakupoglu A, Giray S, Unal OF and Duren M: Intraoperative ultrasonography is useful in surgical management of neck metastases in differentiated thyroid cancers. Endocrine 48: 248-253, 2015.

19. Matsuzawa F, Einawa T, Abe H, Suzuki T, Hamaguchi J, Kaga T, Sato M, Oomura M, Takata Y, Fujibe A, et al: Accurate diagnosis of axillary lymph node metastasis using contrast-enhanced ultrasonography with Sonazoid. Mol Clin Oncol 3: 299-302, 2015.

20. Matsuzawa F, Omoto K, Einawa T, Suzuki T, Hamaguchi J, Kaga T, Sato M, Oomura M, Takata Y, Fujibe A, et al: Accurate evaluation of axillary sentinel lymph node metastasis using contrast-enhanced ultrasonography with Sonazoid in breast cancer: A preliminary clinical trial. Springerplus 4: 509, 2015.

21. Yu M, Liu Q, Song HP, Han ZH, Su HL, He GB and Zhou XD: Clinical application of contrast-enhanced ultrasonography in diagnosis of superficial lymphadenopathy. J Ultrasound Med 29: 735-740, 2010.

22. Poanta L, Serban O, Pascu I, Pop S, Cosgarea M and Fodor D: The place of CEUS in distinguishing benign from malignant cervical lymph nodes: A prospective study. Med Ultrason 16: 7-14, 2014.

This work is licensed under a Creative Commons Attribution-NonCommercial-NoDerivatives 4.0 International (CC BY-NC-ND 4.0) License. 